\title{
The Decay Rate of Orthopositronium
}

\author{
J. S. Nico, D. W. Gidley, and A. Rich \\ Department of Physics \\ University of Michigan \\ Ann Arbor, MI 48109 \\ P. W. Zitzewitz \\ Department of Natural Sciences \\ University of Michigan - Dearborn \\ Dearborn, MI 48128
}

\begin{abstract}
We review recent measurements of the orthopositronium decay rate, $\lambda_{T}$, and present results of a new 230-ppm measurement using the vacuum technique. It corroborates, at the 6.2 sigma level, the discrepancy between theory and a recent 200-ppm measurement of $\lambda_{T}$ in gases.

\section{Introduction}

Quantum electrodynamics (QED) has been rigorously tested in bound-state systems of atomic hydrogen and heavier atoms, in muonium, and in positronium. Positronium (Ps), the positronelectron bound state, and muonium, the bound state of the positive muon and the electron, offer the advantages of being purely leptonic systems, with the electromagnetic interaction being the only interaction present at current levels of experimental and calculational precision. In muonium the ratios of the mass and magnetic moment relative to the electron are needed to permit precision comparisons with QED, while in Ps these ratios are equal to one both experimentally and theoretically (CPT invariance). Positronium, being a low-mass particle-antiparticle system, has the additional feature of being formulated in a relativistic 2-body equation as embodied by the Bethe-Salpeter equation. It has virtual and real annihilation terms that can be tested. The annihilation of the ground state into two or more photons provides an interesting and unique test of QED. In fact, the most rigorous checks on any decay
\end{abstract}


rate calculations are the precision measurements of the Ps singlet $\left({ }^{1} \mathrm{~S}_{0}\right)$ and triplet $\left({ }^{3} \mathrm{~S}_{1}\right)$ decay rates. The decay of the triplet state, or orthopositronium (o-Ps), is more easily measured with high precision because its $140 \mathrm{~ns}$ lifetime is $10^{3}$ times longer than that of the singlet state. Therefore, after a brief discussion of the theoretical calculations and prior experimental results, we will focus on our most recent determination of the triplet decay rate.

The $o$-Ps decay rate, $\lambda_{T}$, is expressed as a sum of its decay rates into three photons $\left(\lambda_{3}\right)$, five photons $\left(\lambda_{5}\right)$, etc.:

$$
\lambda_{T}=\lambda_{3}+\lambda_{5}+\lambda_{7}+\ldots
$$

The effect of the five photon decay has been calculated ${ }^{1}$ and found to be of order $10^{-6} \lambda_{3}$. Thus, to sufficient precision, $\lambda_{T}$ is given by $\lambda_{3}$ where

$$
\lambda_{3}=\alpha^{6} \frac{m c^{2}}{\hbar} \frac{2\left(\pi^{2}-9\right)}{9 \pi}\left[1+A\left(\frac{\alpha}{\pi}\right)+\frac{1}{3} \alpha^{2} \ln \alpha+B\left(\frac{\alpha}{\pi}\right)^{2}+\ldots\right]
$$

The coefficient $A$ has been calculated independently yielding values of $A=-10.266(11)^{2}$ and $A=-10.282(3)^{3}$ Using the more precise value of $A$ from Ref. 3 , one obtains $\lambda_{3}=7.03830(7) \mu \mathrm{s}^{-1}$. We note that $B$ is still uncalculated, and if it is of order 10 , the second order term could contribute $50 \mathrm{ppm}$, or $0.00035 \mu \mathrm{s}^{-1}$.

The most recent precision measurements of $\lambda_{T}$ are shown in Table I. Decay rate experiments are divided into either gas or vacuum type according to the method of o-Ps formation. In gas experiments positrons from a radioactive source (typically $200 \mathrm{keV}$ energy) are stopped in a gas and form o-Ps with roughly $25 \%$ efficiency. The thermalized positronium remains near its formation site, diffusing less than a millimeter, and decays into three gamma rays. The measured decay rate is always increased due to collisional "pickoff" quenching of $o$-Ps with the gas atoms. The vacuum decay rate $\lambda_{T}$ is obtained by a roughly $1 \%$ extrapolation to zero gas density. 
In vacuum experiments, positrons from a low-energy (typically $1 \mathrm{keV}$ ) positron beam strike a target surface where up to $30 \%$ are expelled from the surface into the vacuum as o-Ps. The positronium is emitted at energies typically of a few $\mathrm{eV}$ and must be confined within view of the gamma detectors by a confinement cavity. The extrapolation required to remove systematic effects of this cavity is well under $0.1 \%$, and thus this experiment is virtually a direct measurement of the vacuum decay rate. It is, however, subject to very different systematic effects than the gas experiment, as discussed below.

\begin{tabular}{|ccc|}
\hline$\lambda_{T}\left(\mu \mathrm{s}^{-1}\right)$ & Technique & Date \\
\hline $7.056 \pm 0.007$ & gas & 1978, Ref. 4 \\
$7.045 \pm 0.006$ & gas & 1978, Ref. 5 \\
$7.050 \pm 0.013$ & vacuum & 1978, Ref. 6 \\
$7.051 \pm 0.005$ & gas & 1982, Ref. 7 \\
$7.0514 \pm 0.0014$ & gas & 1987, Ref. 8 \\
$7.031 \pm 0.007$ & vacuum & 1987, Ref. 9 \\
\hline
\end{tabular}

Table I. Recent measurements of $\lambda_{T}$.

The result of the most recent experiment performed in gas, shown in Table $I$, is a factor of four more precise (at $200 \mathrm{ppm}$ ) than previous measurements. It is 9.4 experimental standard deviations higher than the theoretical value. A recent vacuum measurement, however, found at the 1000-ppm level a value one sigma below theory and three sigma below the gas result. Neither it nor the earlier 1978 vacuum measurement is precise enough to check rigorously the 9.4 sigma discrepancy. Such a check would be very attractive because, as noted above, the vacuum measurements are systematically so different from the gas measurement: there is no collisional gas quenching (nor any possible Ps-gas chemistry) and hence the extrapolation needed to extract $\lambda_{T}$ is almost negligible compared to that required in gases; the kinetic energy and mechanism of Ps formation is different; and there is no applied magnetic field.

\section{Experimental Method and Systematic Effects}

We now turn to a discussion of our new measurement ${ }^{10}$ of $\lambda_{T}$ using a systematically improved vacuum technique. It employs a 
slow positron beam to form positronium in an evacuated $\left(10^{-9}\right.$ Torr), MgO-lined cavity, similar to the technique used in Ref. 6 . Several improvements in beam technology have been utilized; in particular, remoderation ${ }^{11,12}$, beam gating, and time-tagging ${ }^{13}$. The apparatus, shown in Fig. 1, is divided into two sections: a primary beam that moderates and focuses the positrons from a $50 \mathrm{mCi}{ }^{22} \mathrm{Na}$ source onto a $\mathrm{Ni}$ foil remoderator, and a secondary beam that delivers the remoderated positrons into the positronium formation cavity. Secondary electrons produced when the primary beam strikes the $\mathrm{Ni}$ foil are detected by a channel electron multiplier array (CEMA) and serve as the start signal for the timing electronics. This time-tagging of the beam eliminates systematics associated with a start detector inside the Ps formation cavity ${ }^{6,9}$. Since the tagging efficiency is only $15 \%$, it is necessary to use the start signal to trigger the opening of a normally-closed gate in order to eliminate noise from untagged positrons. The gated and timetagged positrons are focused into an aluminum cavity and strike the fumed $\mathrm{MgO}$ layer at $700 \mathrm{eV}$. The $\mathrm{MgO}$, in addition to being an efficient $o$-Ps formation surface $(\sim 25 \%)$, minimizes collisional quenching at the cavity walls. The annihilation gammas are detected by two semi-annular fast-plastic scintillators that surround the cavity. These detectors are surrounded with $\mathrm{Pb}$ shielding so that the effects of ungated positrons and gated but poorly focused positrons are negligible. Each scintillator is coupled by a light pipe to two RCA 8852 PMTs, and the output signals of the four PMTs are mixed to comprise the stop signal.

The lifetime spectrum consists of a histogram of CEMA-start to gamma-stop time interval measurements in the range from 0 to $2 \mu \mathrm{s}$ (for further details see Ref. 10). Approximately $7 \times 10^{7}$ o-Ps events were collected in a 2-day run, and the spectrum for a particular cavity is typically the sum of 8-10 such runs. Beginning $100 \mathrm{~ns}$ beyond the prompt peak the spectrum is fitted by a maximumlikelihood program to a function of the form

$$
N(t)=\left(A e^{-\lambda t}+B\right) e^{-R t}
$$

where $A, B$, and $\lambda$ are the fitted parameters and $R$ is the measured 


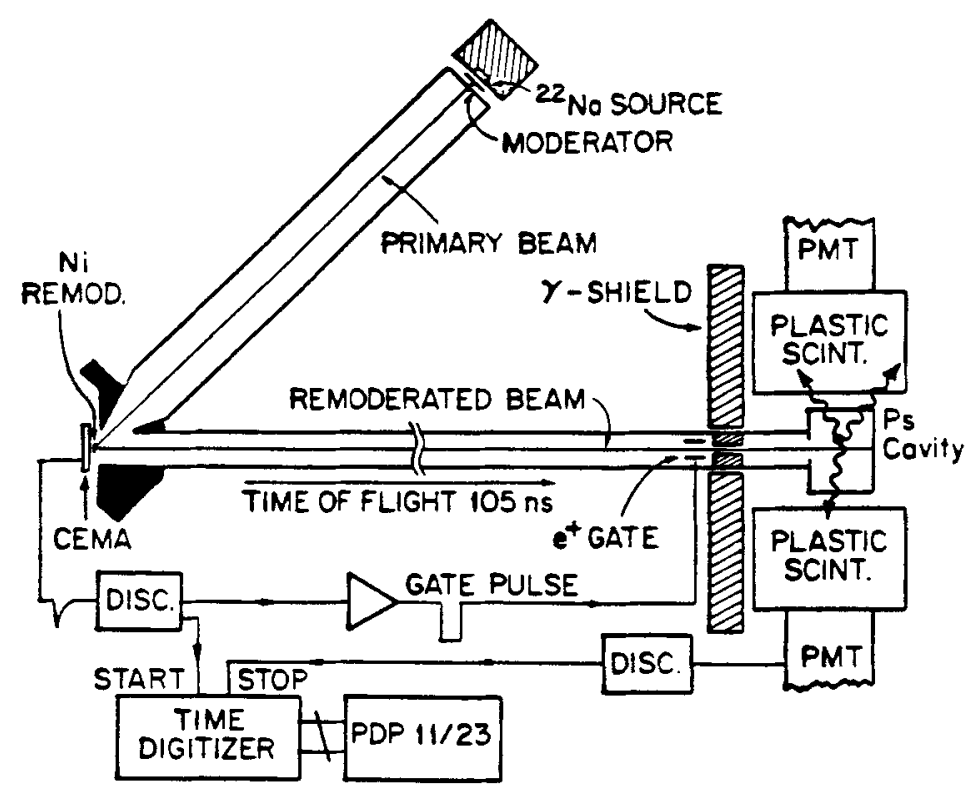

Figure 1. Time-tagged and gated slow positron beam used to measure the o-Ps decay rate.

background rate ( $\sim 200 \mathrm{cps}$ ) during the gate-closed (beam off) interval. The signal-to-noise ratio, $A / B$, is $150-200$ for all spectra. Successively stepping out the start time, $t_{0}$, of the fitting program in $50 \mathrm{~ns}$ intervals and fitting the remaining channels to Eq. (3) reveals a $0.5-1.0 \%$ drop in $\lambda$ as it asymptotically approaches a constant value at $t_{0}=400-450 \mathrm{~ns}$. Thus, at least one additional lifetime component is present in the histogram. The data were therefore refitted to a modification of Eq. (3) that included a second exponential component. We typically obtained fits with acceptable $\chi^{2}$ beginning at $t_{0}=170 \mathrm{~ns}$ and with fitted values of $\lambda$ statistically consistent with the asymptotic value from the single exponential fits. The origin of this additional component with a lifetime of roughly 40 ns is not understood. It does not depend directly on any cavity shape parameter, and its intensity is too large to be attributed to the production of excited states of Ps (discussed below). Although it does not appear in gas decay rate measurements ${ }^{8}$, it is seen in dense powder measurements ${ }^{14}$. 
The most reasonable explanation that appears to satisfy all the observations is that several percent of the o-Ps is formed in small $(\sim 100 \AA)$ pores in the $\mathrm{MgO}$ powder. In this, as well as virtually all alternative explanations, the asymptotic value of $\lambda$ is interpreted as the correct value of $\lambda$ to be associated with that particular cavity.

The asymptotic value of the fitted decay rate is assumed to depend on $\lambda_{T}$ and on terms that account for the rate of disappearance of $o$-Ps through the cavity entrance aperture and the rate of annihilation due to collisions with the cavity walls. Thus,

$$
\lambda=\lambda_{T}+c \frac{\bar{v}}{4} k_{1} k_{2} \frac{A}{V}+\frac{\bar{v}}{4} P_{a} \frac{S}{V}
$$

where $V$ and $S$ are the cavity volume and surface area, respectively, $A$ is the area of the cavity entrance aperture through which the positrons are focused, $\bar{v}$ is the average $o$-Ps velocity, $c$ is the probability that $\gamma$-rays from escaped $o$-Ps will not be detected, and $P_{a}$ is the probability of annihilation during a wall encounter. The disappearance term contains the "Clausing parameter" ${ }^{15}, k_{1}$, that accounts for reduced o-Ps disappearance through an aperture of nonzero thickness and the parameter $k_{2}$ that accounts for the nonuniform particle density that occurs when the aperture diameter is nearly as large as the $o-P s$ mean free path in the cavity ${ }^{16}$. The vacuum decay rate can be obtained from Eq. (4) as a 2-variable extrapolation to the intercept of a plane in $\frac{A}{V}$ and $\frac{S}{V}$ (see Fig. 2). Five different cavities with $\frac{S}{V}$ ratios in the range $0.8-6.6 \mathrm{~cm}^{-1}$ are used with two or three different aperture diameters. The intercept of Eq. (4) is $7.0497 \pm 0.0013 \mu \mathrm{s}^{-1}$. Note that the measured $\lambda$ are within one standard deviation of this intercept, permitting an extrapolation that is almost 2 orders of magnitude smaller than that encountered in gas experiments.

Eq. (4) contains the assumption that the slope of the $\frac{A}{V}$ disappearance term will be the same for all cavities. In fact, we see a decrease in this slope at the 1-2 sigma level as $\frac{S}{V}$ increases. We removed this slope restriction by refitting the data for each cavity to Eq. (4) without the $\frac{S}{V}$ term. The $\frac{A}{V}=0$ intercepts for each 


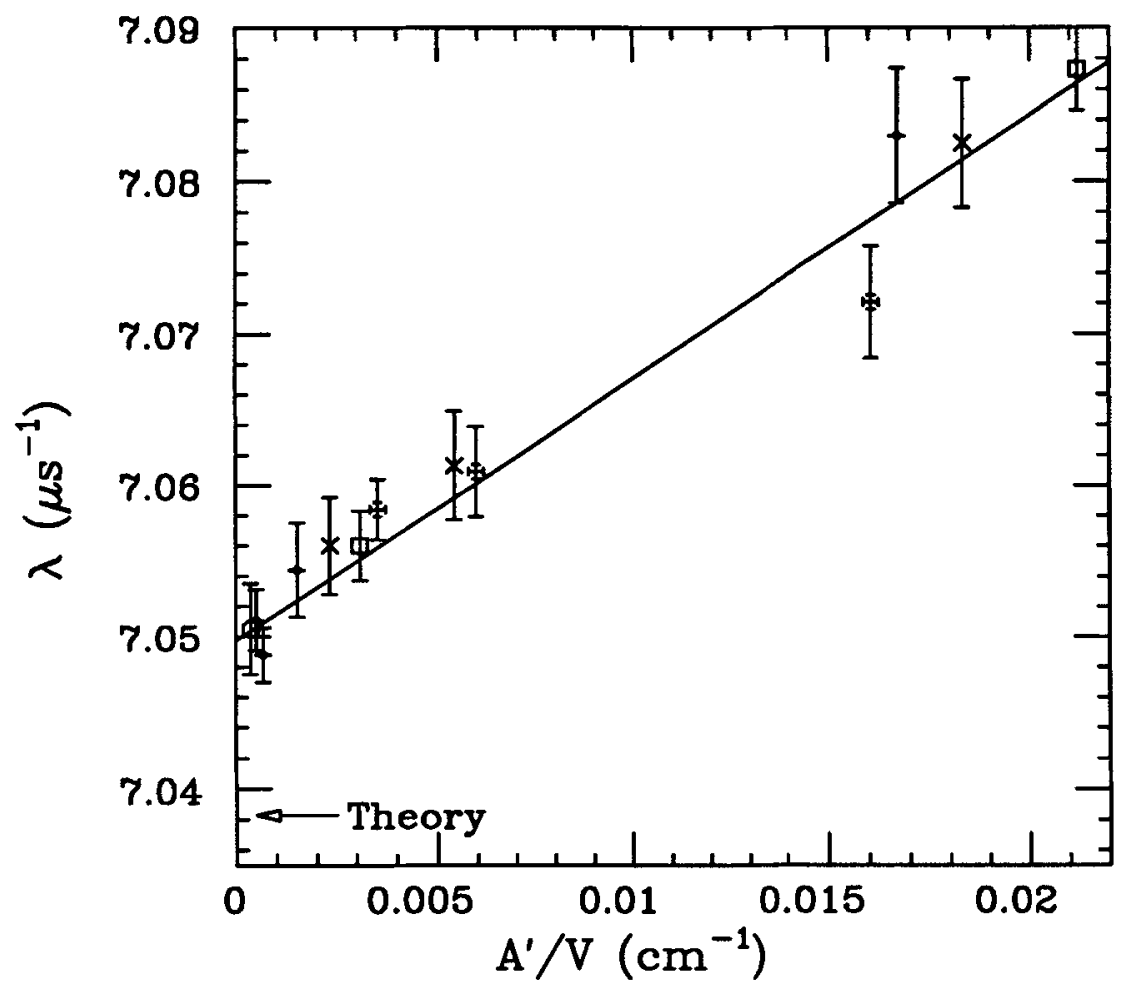

Figure 2. The fitted decay rate as a function of $\frac{A^{\prime}}{V}$ (where $A^{\prime}=k_{1} k_{2} A$ ) for all cavity geometries. Plotting symbols distinguish the $\frac{S}{V}$ values as shown in Fig. 3. The solid line is the intersection of the plane determined by the fit to Eq. (4) with the $\frac{S}{V}=0$ plane. The $\chi^{2}$ of the fit is only 2 for 10 degrees of freedom, indicating a conservative error bar assignment on the asymptotic value of $\lambda$ in Eq. (3).

cavity are then plotted versus $\frac{S}{V}$ as shown in Fig. 3. Extrapolation to $\frac{S}{V}=0$ yields $\lambda_{T}=7.0482 \pm 0.0015 \mu \mathrm{s}^{-1}$, or about one sigma lower than the 2-variable extrapolation. From the slope of the $\frac{S}{V}$ collisional quenching term, $0.0009 \pm 0.0005 \mathrm{~cm} / \mu$ s (see Fig. 3), we obtain $P_{a} \approx 5 \times 10^{-5}$, assuming $o$-Ps with an average energy of $3 \mathrm{eV}^{14,17}$. We cannot account for $P_{a}$ or the decrease in the $\frac{A}{V}$ slope unless Ps makes at least $10 \mathrm{MgO}$ grain collisions per cavity 
The Decay Rate of Orthopositronium

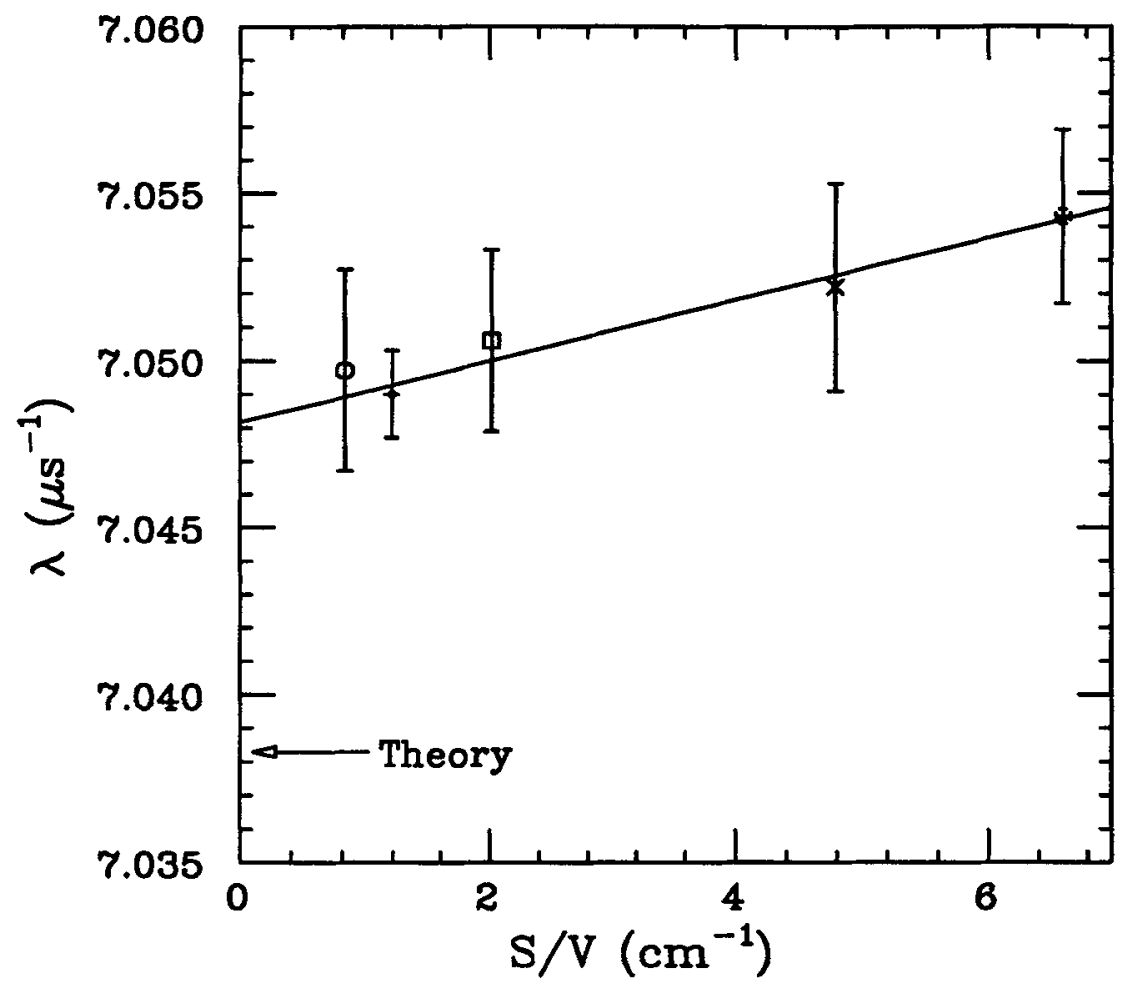

Figure 3. Results of the individual extrapolations to $\frac{A^{\prime}}{V}=0$ are shown for each cavity as a function of $\frac{S}{V}$. The solid line represents a linear extrapolation to $\frac{S}{V}=0$ as our determination of $\lambda_{T}$. The $\chi^{2}$ of the fit is 0.2 for 3 degrees of freedom.

wall encounter. Nevertheless, we select the result of this doubleextrapolation as our determination of $\lambda_{T}$ since it is the most free of restrictions on the fitted parameters.

We return now to systematic effects that might alter the shape of the annihilation spectrum as given by Eq. (3) and hence shift our determination of $\lambda_{T}$. A major systematic uncertainty that limited the 1978 experiment is related to the possibility that some of the Ps does not diffuse out of the $\mathrm{MgO}$ powder (where the decay rate in the open, intergranular space is roughly $2.5 \%$ higher than $\left.\lambda_{T}{ }^{14}\right)$. A component with a decay rate that close to $\lambda_{T}$ would go undetected as we stepped out the start time, $t_{0}$, of the fitting 


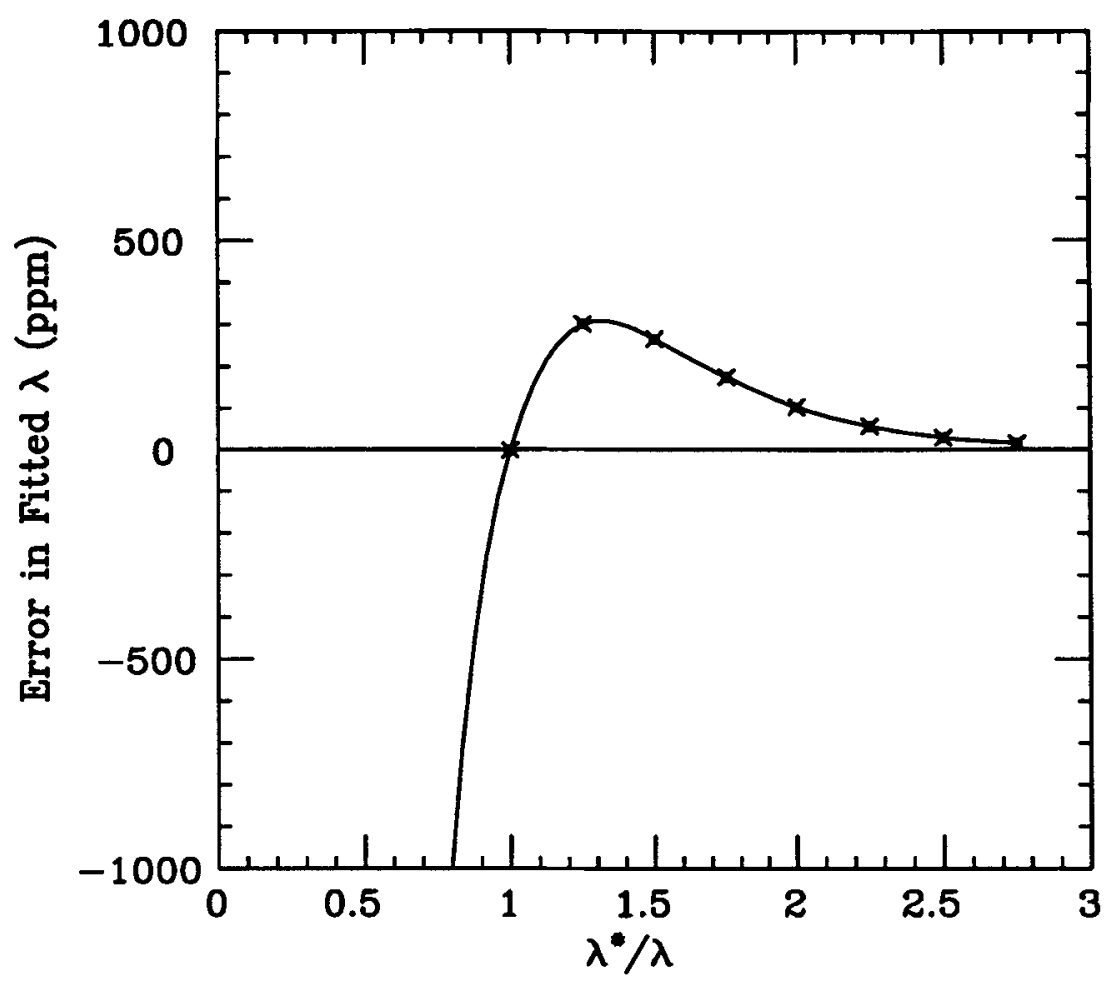

Figure 4. Error in $\lambda_{T}$ vs. the possible decay rates of the $2^{3} S_{1}$ state at a fixed intensity of $0.3 \%$.

program, but it would shift $\lambda$ upwards. To search for this effect, a lead gamma ray collimator was constructed to view only the gamma rays originating at or very near the formation surface. We have set a limit on a quantity $f_{M g O}$, the fraction of $o$-Ps beyond $300 \mathrm{~ns}$ that decays in the formation powder, of $f_{M g O} \leq 0.3 \%$. Its effect on the fitted value of $\lambda$ is then limited to $(2.5 \%) f_{M g O}$, or 80 ppm.

The formation of excited states of positronium $\left(\mathrm{Ps}^{*}\right)$ can also add lifetime components to Eq. (3). The $2{ }^{3} \mathrm{~S}_{1}$ state is the only one with a formation rate and vacuum lifetime $(1.1 \mu \mathrm{s})$ that could possibly affect the fitted value of $\lambda_{T}$. Using the $n=2$ detection apparatus at Michigan ${ }^{18}$, we have measured the production fraction of $2^{3} \mathrm{~S}_{1}$ relative to $1^{3} \mathrm{~S}_{1}$ in $\mathrm{MgO}$ to be $3 \times 10^{-3}$. The results of fitting computer generated spectra, Fig. 4, show the effect of 
such a component with $0.3 \%$ intensity and any decay rate. The $2^{3} \mathrm{~S}_{1}$ lifetime must be quenched to $70-130 \mathrm{~ns}$ in order to produce a $100-300 \mathrm{ppm}$ increase in the fitted value of $\lambda_{T}$, or to $145-200$ ns to decrease $\lambda_{T}$ by $100-1500 \mathrm{ppm}$. (In all cavities, the applied electric fields of at least $150 \mathrm{~V} / \mathrm{cm}$ Stark-quench the $2^{3} \mathrm{~S}_{1}$ lifetime to $200 \mathrm{~ns}$ or less.) The probability that $2^{3} \mathrm{~S}_{1}$ Ps have lifetimes that long is considered to be unlikely based on a previous experiment ${ }^{8}$ that determined the cross section for collisional de-excitation of $2^{3} \mathrm{~S}_{1}$ states in gases to be of order $10^{-14} \mathrm{~cm}^{2}$. It is improbable that the $2^{3} \mathrm{~S}_{1}$ state will survive beyond the first collision with an $\mathrm{MgO}$ grain, which occurs within $50 \mathrm{~ns}$ in a typical cavity. In view of these considerations, we conclude that the effect of excited state Ps for $t_{0}>400 \mathrm{~ns}$ is negligible.

\section{Conclusion}

Using the extrapolation in Fig. 3, our result for the $1^{3} \mathrm{~S}_{1}$ decay rate is $\lambda_{T}=7.0482 \pm 0.0016 \mu \mathrm{s}^{-1}(230 \mathrm{ppm})$. The dominant contributions to the uncertainty are the $210-\mathrm{ppm}$ statistical error (that includes the uncertainties in the $\frac{A}{V}$ and $\frac{S}{V}$ extrapolations), the 80 -ppm $\mathrm{MgO}$ powder systematic, and the time calibration uncertainty of $40 \mathrm{ppm}$. Fig. 5 summarizes the status of the measurements of $\lambda_{T}$ given in Table I and includes our new vacuum result. The value does not substantiate the low decay rate reported in Ref. 9. It is 6.2 sigma above theory and 1.5 sigma below the gas measurement of Ref. 8. Although a 1.5 sigma difference is only marginally significant, if the four different gas extrapolations of Ref. 8 are considered individually, then the present vacuum result favors the lower decay rates measured in $\mathrm{N}_{2}$ and Ne. The higher values measured in isobutane and neopentane resulted in the four values as a whole being slightly over-dispersive. This suggests that the extrapolation to zero density in the hydrocarbon gases (which have large virial coefficients, low liquefaction pressures, and strong positron interactions) may be more complicated than in the more noble gases $\mathrm{Ne}$ and $\mathrm{N}_{2}$. The determination of $\lambda_{T}$ based on only these two gases would be $7.0492 \pm 0.0015 \mu \mathrm{s}^{-1}$, in good agreement with the present measurement.

Decay modes of o-Ps not included in QED calculations, such 


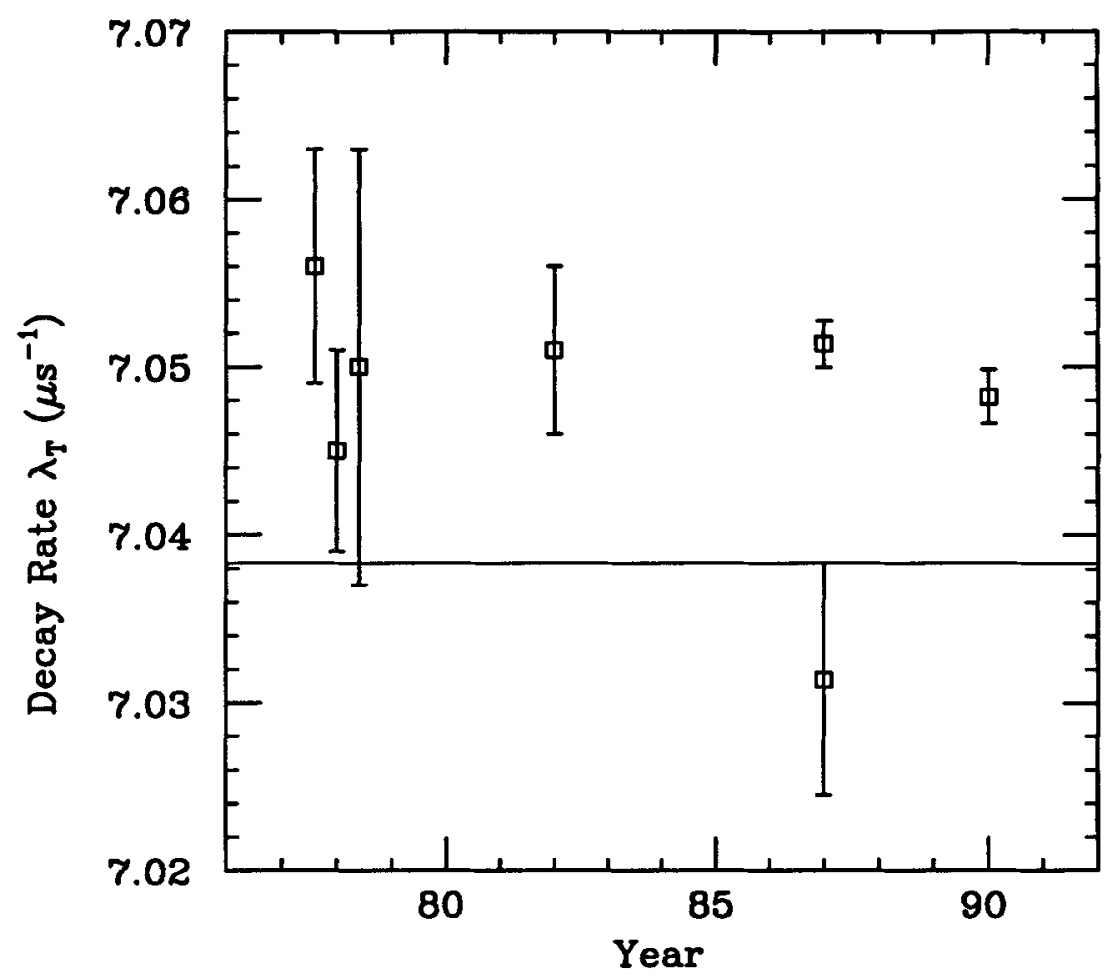

Figure 5. Chronology of recent o-Ps decay rate measurements. The solid line indicates the theoretical value.

as zero, one, or two photon annihilation, have been considered in a number of experiments. The annihilation into zero photons, i.e., $o$-Ps $\rightarrow$ noninteracting particles, was ruled out at the $350-\mathrm{ppm}$ level ${ }^{19}$. Several groups searched for the decay into one photon accompanied by a particle of mass $m_{x}$ greater than $100 \mathrm{keV}$ producing null results at the $30-\mathrm{ppm}$ level ${ }^{20}$. A recent experiment looked at the same process for particles in the mass region below $100 \mathrm{keV}^{21}$. They quote upper limits of $64 \mathrm{ppm}$ for $m_{x}$ below $30 \mathrm{keV}$ and $7.6 \mathrm{ppm}$ for masses around $100 \mathrm{keV}$; these limits are about 2 orders of magnitude below the branching ratio needed to resolve the difference between the new experiment and theory. Recently, decay of $o$-Ps into two gamma rays has been investigated ${ }^{22}$ and the decay into fractionally charged particles has been hypothesized ${ }^{23}$. In par- 
ticular, it is suggested that ${ }^{3} \mathrm{~S}_{1} \rightarrow \epsilon \bar{\epsilon}$ where $\epsilon$ is a light $\left(m_{\epsilon}<m_{e}\right)$ particle with electric charge $Q_{\epsilon}=q e$ and $q$ is of order $10^{-3}$. No experiment has as yet been performed to search directly for this decay mode. Insofar as the $\epsilon$ decay products are noninteracting particles, this branching ratio should be limited to $350 \mathrm{ppm}^{19}$. Thus, exotic decay modes do not appear to be viable explanations. We do note that a value of the order $\left(\frac{\alpha}{\pi}\right)^{2}$ coefficient, $B$ in Eq. (2), equal to $250 \pm 40$ would resolve the disagreement. A coefficient that large cannot be ruled out a priori, and hence calculation of $B$ is crucial in order to fully confront QED theory at the level of accuracy afforded by the experiments.

We thank Michael Bretz, Ralph Conti, G.W. Ford, William Frieze, Lisa Lapidus, Aaron Noble, Norman Ramsey, Tom Steiger, Chris Westbrook, and members of the Michigan positron group for helpful discussions. We thank Tom Steiger for measurements of the $n=2$ excited state formation rates. This work is supported by the National Science Foundation under grants PHY8803718 and PHY8818582 and by the Office of the Vice-President for Research of the University of Michigan. 


\section{References}

1. G. S. Adkins and F. R. Brown, Phys. Rev. A 28, 1164 (1983); G. P. Lepage, P. B. Mackenzie, K. H. Streng, and P. M. Zerwas, Phys Rev A 28, 3090 (1983).

2. W. G. Caswell and G. P. Lepage, Phys Rev. A 20, 36 (1979).

3. G. S. Adkins, Ann. Phys. (N.Y.) 146, 78 (1983).

4. D. W. Gidley, A. Rich, P. W. Zitzewitz, and D. A. L. Paul, Phys. Rev. Lett. 40, 737 (1978).

5. T. C. Griffith, G. R. Heyland, K. S. Lines, and T. R. Twomey, J. Phys. B 11, 743 (1978).

6. D. W. Gidley and P. W. Zitzewitz, Phys. Lett. 69A, 97 (1978).

7. D. W. Gidley, A. Rich, E. Sweetman, and D. West, Phys. Rev. Lett. 49, 525 (1982).

8. C. I. Westbrook, D. W. Gidley, R. S. Conti, and A. Rich, Phys. Rev. A 40, 5489 (1989).

9. P. Hasbach, G. Hilkert, E. Klempt, and G. Werth, Nuovo Cimento 97 A, 419 (1987).

10. J. S. Nico, D. W. Gidley, A. Rich, and P. W. Zitzewitz, Phys. Rev. Lett. 65, 1344 (1990).

11. A. P. Mills Jr., Appl. Phys 23, 189 (1980).

12. W. E. Frieze, D. W. Gidley, and K. G. Lynn, Phys Rev B 31, 5628 (1985).

13. J. Van House, A. Rich, and P. W. Zitzewitz, Orig. of Life 14, 413 (1984); J. Van House, Ph.D. Thesis, The University of Michigan, Ann Arbor, 1984.

14. D. W. Gidley, Ph.D. Thesis, The University of Michigan, Ann Arbor, 1979.

15. P. Clausing, Ann. Physik 12, 961 (1932).

16. M. Knudsen, Ann. Physik 28, 75, 999 (1909); 35, 389 (1911).

17. P. Sferlazzo, S. Berko, and K. F. Canter, Phys. Rev. B 35, 5315 (1987).

18. S. Hatamian, R. S. Conti, and A. Rich, Phys. Rev. Lett. 58, 1833 (1987).

19. G. S. Atoyan, S. N. Gninenko, V. I. Razin, and Yu. V. Ryabov, Phys. Lett. 220 B, 317 (1989). 
494 The Decay Rate of Orthopositronium

20. G. Carboni and W. Dahme, Phys Lett. 123B 349, (1983); U. Amaldi, G. Carboni, B. Jonson, and J. Thun, Phys. Lett. 153B, 444 (1985); V. Metag et al., Nucl. Phys. A409, 331 (1983).

21. S. Orito, K. Yoshimura, T. Haga, M. Minowa, and M. Tsuchiaki, Phys. Rev. Lett. 63, 597 (1989).

22. A. P. Mills, Jr. and D. M. Zuckerman, Phys. Rev. Lett. 64, 2637 (1990).

23. M. I. Dobroliubov and A. Yu. Ignatiev, Phys. Rev. Lett. 65, 679 (1990). 